\title{
Thyroid axis function after in-patient treatment of acute psychosis with antipsychotics: a naturalistic study
}

Robertas Bunevicius ${ }^{1}$, Vesta Steibliene ${ }^{2^{*}}$ and Arthur J Prange $\mathrm{Jr}^{3}$

\begin{abstract}
Background: Endocrine function in psychiatric patients may be affected by mental disorder itself as well as by antipsychotic medications.

The aim of this naturalistic observational study was to determine if treatment of acute psychotic episode with antipsychotic medication affects thyroid axis hormone concentrations and if such changes are associated with symptomatic improvement.
\end{abstract}

Methods: Eighty six adult acute psychotic patients, consecutively admitted to a mental hospital, were recruited for the study. All patients were physically healthy and without thyroid disease. During the hospitalization period all study patients received treatment with antipsychotic medication according to clinical need. Severity of the psychotic episode was evaluated using the Brief Psychiatric Rating Scale (BPRS) and venous blood samples were drawn for analysis of free triiodothyronine (FT3), free thyroxine (FT4), and thyroid stimulating hormone (TSH) concentrations on the day of admission and on the day of discharge from the hospital.

Results: Antipsychotic drug treatment was associated with decrease of mean FT3 $(p<0.001)$ and FT4 $(p=0.002)$ concentrations; and with increase of mean TSH ( $p=0.016)$ concentrations. Changes in thyroid hormone concentrations were mostly predicted by baseline hormone concentrations. Individual changes were not limited to decrease in high hormone concentrations; in patients who had low FT3 or FT4 concentrations, treatment resulted in increase in concentrations. Such an increase was established in one-quarter of patients for FT3 concentrations and in one-third of patients for FT4 concentrations. Fall in FT4 concentrations negatively correlated with the improvement in the BPRS score $(r=-0.235, p=0.023)$.

Conclusions: The study indicates that antipsychotic treatment resulted in a decrease in mean FT3 concentrations and in an increase in mean TSH concentrations after recovery from acute psychosis. Symptomatic improvement was less evident in patients who experienced a decrease in FT4 concentrations.

Trial registration: EudraCT No.2007-001541-18

Keywords: Psychosis, Thyroxine, Triiodothyronine, Thyroid stimulating hormone, Treatment, Antipsychotics

\footnotetext{
* Correspondence: vsteibliene@mail.It

${ }^{2}$ Clinic of Psychiatry, Lithuanian University of Health Sciences, Mickeviciaus str. 9, Kaunas LT-44307, Lithuania

Full list of author information is available at the end of the article
} 


\section{Background}

Acute psychoses, including schizophrenia, have a life time prevalence of $2-3 \%$ in the general population [1]. Patients suffer decrease in social functioning and quality of life, and society suffers a huge economic burden [2].

Endocrine function in psychiatric patients may be affected by mental disorder itself as well as by medications. Neuro-endocrine abnormalities have received significantly less attention in psychotic disorders relative to affective disorders [3]. In acute psychotic episodes [4] as well as in chronic schizophrenia $[5,6]$ changes in thyroid function and thyroid autoimmunity have been reported. Elevated serum thyroxine (T4) concentrations with or without decrease in triiodothyronine (T3) concentrations have been found in acute schizophrenic patients [7] as well as in patients with other severe mental disorders, such as acute major depression and mania [8-10]. In our recent cross-sectional study [11], we have confirmed that patients with acute psychosis upon admission to the hospital had elevated T4 concentrations, especially patients who were free from treatment with antipsychotics. This syndrome of elevated T4 concentrations in acutely psychotic patients is coined as transient hyperthyroxinemia because it usually resolves during recovery from a psychotic episode [12,13]. However, the majority of schizophrenic patients appear to be euthyroid with normal thyroid stimulating hormone (TSH) concentration and normal TSH response to thyrotropin-releasing hormone (TRH) challenge [14,15].

Studies examining the possible effects of antipsychotic treatment on thyroid axis function in psychotic patients are limited to reports on recuperation from the hyperthyroxinemia [16-18]. Data on the effects of antipsychotic treatments on T3 and TSH concentrations are scant and data on the associations between changes in thyroid hormone concentrations and mental status are lacking.

In the light of the above observations, we have set out a naturalistic study in acute psychotic patients aiming to determine (1) if antipsychotic treatment affects thyroid axis hormone concentrations and (2) if changes in thyroid axis hormone concentrations after antipsychotic treatment are associated with symptomatic improvement.

\section{Methods}

\section{Study population}

During a two year period adult acute psychotic patients, consecutively admitted to the acute psychosis department of the District Mental Hospital in Kaunas, Lithuania, were screened for eligibility to participate in the prospective interventional study (EudraCT No. 2007-001541-18; study results not published). Patients not meeting the interventional study criteria were invited to participate in this prospective naturalistic observational study if they were able to understand the study aims and procedures. The protocol and informed consent form of the study were approved by the Lithuanian Bioethics Committee and each study patient had signed a written informed consent form.

One hundred and four psychotic patients aged from 18 to 70 years old with no significant or unstable medical conditions, and physically healthy based on the physical examination, past medical history, and results of routine blood and urine tests, were invited to participate in the study. Exclusion criteria for the study were: any significant and/or unstable somatic condition, a history of epileptic seizures, suicidal tendencies or severe agitation, a history of substance or drug dependence within 6 month of the screening visit, treatment for concurrent somatic condition during the psychosis treatment period, treatment with long-acting antipsychotics during the four month period before the hospital admission, or electroconvulsive therapy during the three month period before the admission. Eight patients were excluded from the study due to thyroid dysfunction at baseline: six patients had TSH concentrations higher than $4.05 \mu \mathrm{IU} / \mathrm{ml}$ suggesting hypothyroidism and two patients had TSH concentration lower than $0.17 \mu \mathrm{IU} / \mathrm{ml}$ suggesting hyperthyroidism. We have also excluded seven patients who had thyroid peroxidase antibody (TPOAb) concentrations higher than $20 \mathrm{IU} / \mathrm{ml}$, suggesting possible autoimmune thyroid disease. Furthermore, three patients did not receive treatment with antipsychotic medications during the hospitalization period and were excluded from the analyses. Therefore, 86 (83\%) acute psychotic patients (43 men and 43 women, mean age 36 years) comprised the study sample and were studied.

Thirty patients were admitted for their first psychotic episode and 56 patients have experienced relapsed psychotic episode. In relapsed patients, the duration since their previous psychotic episode averaged five years. Mean treatment duration for the current psychotic episode was 29 days (range from 4 days to 55 days).

Following a naturalistic design of the study, patients were treated according to the usual clinical practice and there were no restrictions for psychiatric treatment(s) selection. All 86 patients were treated with antipsychotic medication (41 patients with haloperidol, 16 with risperidone, 14 with olanzapine, 5 with quetiapine, 6 with amisulpiride, 3 with ziprazidone, and 1 with tiapride); 83 patients were treated with benzodiazepines (diazepam, clonazepam, or lorazepam); and 25 patients were treated with antidepressants (amitriptyline, sertraline, paroxetine, escitalopram, or venlafaxine). With regards to combined psychiatric treatment, 59 patients received antipsychotic medication and benzodiazepines, 24 patients - antipsychotic medication, antidepressant and benzodiazepines, 2 patients - antipsychotic medication only, and 1 patient antipsychotic medication and antidepressant.

The control group consisted of 120 consecutive blood donors recruited from the Kaunas Blood Donors Center, Lithuania. All blood donors were evaluated according to the 
standard procedures and were included in the study if they were considered as being healthy, with no history of past or present clinical psychiatric disorder or unstable medical condition; and without current use of medication, including psychiatric drugs. Five blood donors with elevated TSH concentration were excluded from the analysis and there were no subjects with suppressed TSH concentrations. We have excluded six blood donors with TPOAb concentrations higher than $20 \mathrm{IU} / \mathrm{ml}$. Therefore blood samples from 109 control subjects (66 men and 43 women, mean age 34 years) were used for biochemical comparisons.

\section{Psychiatric evaluations}

Psychiatric diagnoses were established according to the Diagnostic and Statistical Manual of Mental Disorders, 4th Edition, Text Revision (DSM-IV-TR) diagnostic criteria [19] using the MINI-Plus 5.0.0 structured clinical interview [20] for all study patients but not for the control group. The MINI is designed as a brief structured interview for the diagnoses of major Axis I psychiatric disorders according to the DSM-IV-TR criteria. The MINI-Plus is comparable with other standard diagnostic instruments, such as the Structured Clinical Interview for DSM-IV (SCID) [21] or Composite International Diagnostic Interview (CIDI) [22]. The MINI is a reliable instrument and can be administered faster than the SCID or CIDI. The MINI-Plus is divided into 26 modules, identified by letters, each corresponding to a diagnostic category, pertaining to past and current diagnoses of mental disorders. At the beginning of each diagnostic module (except for the psychotic disorders module), screening questions corresponding to the main diagnostic criteria of the disorder are presented. At the end of each module, a diagnostic box permits the clinician to indicate whether diagnostic criteria for a specific mental disorder have been met.

We have used three modules of the MINI-Plus addressing diagnoses of the major depressive episode (module A), (hypo) manic episode (module D), and psychotic disorders (module M). Patients who met the diagnostic criteria for the current psychotic disorder were included in the study. Diagnostic algorithms for psychotic disorders were used to specify diagnosis of the psychotic disorder. Forty-six patients were diagnosed as having schizophrenia; 18 patients, brief psychotic disorder; 13 patients, schizoaffective disorder; 5 patients, schizophreniform disorder and 4 patients, affective psychosis.

Severity of the psychotic disorder was assessed by using the Brief Psychiatric Rating Scale (BPRS) before and after acute psychosis treatment. The BPRS assesses severity of 18 symptom constructs that are rated from 0 (symptom not present) to 6 (symptom is extremely severe) and includes positive, negative and general psychopathology symptoms. The BPRS is useful in gauging treatment efficacy of psychotic disorders [23]. The BPRS is administered by a clinician with knowledge of psychotic disorders and ability to interpret the constructs used in the assessment. Some items (e.g. mannerism and posturing) are based on the observation of the patient's behavior; other items (e.g. anxiety) involve self-reporting by the patient. Family members can also provide information regarding symptoms. Total BPRS score ranges from 0 to 108. Scores below 10 are considered as normal variations. For treatment efficacy analysis we used the change in the total BPRS scores by subtracting BPRS scores after treatment from BPRS scores before treatment. All diagnostic evaluations were made by a trained psychiatrist (VS).

\section{Endocrine measurements}

Venous blood samples were drawn on two occasions: after overnight fast at 8 A.M. on the morning after admission to the hospital and on the day of discharge. Blood was centrifuged, and serum was frozen for storage at $-40^{\circ} \mathrm{C}$. All samples were analyzed for hormone concentrations simultaneously to avoid inter-assay variability.

Serum free T3 (FT3), free T4 (FT4), TSH, and TPOAb concentrations were assessed by radioimmunoassay, using commercial IMULITE kits (Czech Republic). Sensitivity of the FT3 assay was $0.5 \mathrm{pmol} / \mathrm{l} ; \mathrm{FT}_{4}, 0.4 \mathrm{pmol} / \mathrm{l} ; \mathrm{TSH}$, $0.025 \mu \mathrm{IU} / \mathrm{l}$, and TPOAb, $2 \mathrm{IU} / \mathrm{ml}$. The normal reference interval for FT3 concentrations was 3.5-6.9 pmol/l; for FT4, 12.4-21.2 pmol/l; for TSH, 0.17-4.05 $\mu \mathrm{IU} / \mathrm{ml}$; and for TPOAb, > $20 \mathrm{IU} / \mathrm{ml}$.

\section{Statistical analyses}

Kolmogorov-Smirnov statistics showed normal distribution of all variables; therefore, parametric statistical tests were used. Distributions of age, BPRS scores, and FT4, FT3 and $\mathrm{TSH}$ concentrations were expressed as mean $\pm \mathrm{SD}$.

To evaluate significance of change in hormone concentrations and in BPRS scores before and after treatment with antipsychotics, paired t-tests were used. Change was expressed as difference $(\Delta)$ between values after treatment (on the last day before discharge) minus values at baseline (on hospital admission). To evaluate differences in FT3, FT4 and TSH concentrations in patients before and after treatment versus control group, unpaired t-tests were used. Differences were considered as statistically significant when $\mathrm{p}<0.05$.

Differences in changes of thyroid axis hormone concentrations before versus after the treatment $(\Delta \mathrm{FT} 3, \Delta \mathrm{FT} 4$, $\Delta \mathrm{TSH}$ ) as a function of antidepressant treatment (used versus not used) and antipsychotic class (typical versus atypical) were evaluated by using unpaired t-tests. Possible impact of different antipsychotic medication on thyroid hormone concentrations (1) before treatment, (2) after treatment and (3) before versus after treatment was evaluated using the ANOVA analyses with thyroid hormone 
concentrations as dependent variables and antipsychotic medication as independent variable.

To evaluate associations between pre-treatment FT3, FT4 and TSH concentrations and post-treatment change in respective hormone concentrations $(\Delta \mathrm{FT} 3, \Delta \mathrm{FT} 4, \Delta \mathrm{TSH})$ the univariate and multivariate linear regression models were performed. In multivariate models, gender, age, duration of hospitalization, number of past psychotic episodes, duration of the disease, baseline BPRS scores, antidepressant and benzodiazepine treatments during the hospitalization, antipsychotic used during the hospitalization and upon admission together with baseline hormone concentrations were considered as independent variables and changes in hormone concentrations as dependent variables. Data analyses were performed using the SPSS statistical software (version 17.0).

\section{Results}

Upon admission to the hospital all 86 patients had TSH concentrations within the normal range because such was an inclusion criterion. Nevertheless 10 patients had elevated FT4 concentrations indicating euthyroid hyperthyroxinemia.

There were no statistically significant differences between patients and control group subjects according to age and gender; however, there were significant differences in thyroid axis hormone concentrations. As shown in Table 1, mean FT3 concentrations (not different from controls before treatment) decreased after psychosis treatment with antipsychotics $(\mathrm{p}<0.001)$ and became significantly lower compared to controls $(\mathrm{p}<0.001)$. Mean FT4 concentrations also decreased significantly $(\mathrm{p}=0.002)$ after antipsychotic treatment. However, in contrast to FT3 concentrations, mean FT4 concentrations, being increased before treatment $(p=0.004)$, became not different from controls after treatment.

After treatment with antipsychotics TSH concentrations remained in euthyroid range in $81(94 \%)$ patients, and increased above the normal range $(>4.05 \mu \mathrm{IU} / \mathrm{ml})$ in five patients, suggesting possible hypothyroidism. However, FT3 and FT4 concentrations in those five patients remained within the normal range. In all the study patients mean TSH concentrations increased after treatment $(\mathrm{p}=0.016)$ and remained significantly higher compared to controls $(\mathrm{p}<0.001)$. There were no significant differences in changes of thyroid axis hormones concentrations before versus after the treatment period as a function of antidepressant use and antipsychotic class (Table 2). There were no statistically significant differences in thyroid hormone concentrations before treatment, after treatment and before versus after treatment as a function of different antipsychotic medication (all p-values $\geq 0.34$; data not shown).

We found no significant associations between baseline hormone concentrations and improvement in psychotic symptoms. However, change in FT4 concentrations negatively correlated with change in BPRS score $(r=-0.235$ and $\mathrm{p}=0.023$ ) (Figure 1). There were no significant correlations between changes of thyroid axis hormone concentrations, but changes in TSH concentrations correlated positively with changes in FT3 concentrations $(r=0.297$ and $\mathrm{p}=0.004$ ) (Figure 2).

Univariate linear regression analyses revealed strong associations between baseline hormone concentrations and change in respective hormone concentrations after antipsychotic treatment, with baseline FT3 concentrations and baseline FT4 concentrations accounting for $42 \%$ and $44 \%$ of their change, respectively. An association between baseline TSH concentration and the change in TSH concentration accounted for only $16 \%$ of the variance (Figure 3).

In multivariate linear regression models (Table 3) baseline FT3 concentrations accounted for $48 \%$ of the $\triangle \mathrm{FT} 3$ variance, with gender being the second strongest predictor. Baseline FT4 concentrations accounted for 51\% of the $\triangle \mathrm{FT} 4$ variance, with number of psychotic episodes, baseline BPRS scores, antipsychotic used upon and during hospitalization being other significant factors. Baseline TSH values were the only significant correlates of changes in TSH concentrations.

Effects of treatment with antipsychotics were not limited to decrease in elevated thyroid hormone concentrations. In some patients it resulted in an increase of low hormone concentrations. Such an increase occurred in one-quarter of patients for FT3 concentrations $(n=23)$ and in one-third of patients for FT4 concentrations $(n=29)$ (Figure 4).

Table 1 Psychiatric symptoms and thyroid axis hormone concentrations in acute psychotic patients before and after treatment, and in healthy blood donor controls, Mean \pm SD

\begin{tabular}{|c|c|c|c|c|c|c|}
\hline & \multicolumn{2}{|c|}{ Patients, $\mathrm{N}=86$} & \multirow[t]{2}{*}{$\mathrm{p}$ (paired $t$-test) } & \multirow{2}{*}{$\begin{array}{l}\text { Controls } \\
N=109\end{array}$} & \multicolumn{2}{|l|}{$\mathrm{p}$ (unpaired $t$-test) } \\
\hline & $\begin{array}{l}\text { Before } \\
\text { treatment }\end{array}$ & $\begin{array}{l}\text { After } \\
\text { treatment }\end{array}$ & & & $\begin{array}{l}\text { vs patients } \\
\text { before treatment }\end{array}$ & $\begin{array}{l}\text { vs patients } \\
\text { after treatment }\end{array}$ \\
\hline Brief Psychiatric Rating Scale & $39 \pm 8$ & $19 \pm 6$ & $p<0.001$ & - & - & - \\
\hline Free triiodothyronine (pmol/l) & $5.2 \pm 1.0$ & $4.5 \pm 1.0$ & $p<0.001$ & $5.2 \pm 0.9$ & 0.70 & $<0.001$ \\
\hline Free thyroxine $(\mathrm{pmol} / \mathrm{l})$ & $17.9 \pm 3.1$ & $16.8 \pm 2.6$ & $p=0.002$ & $16.8 \pm 2.2$ & 0.004 & 0.89 \\
\hline Thyroid stimulating hormone $(\mu \mathrm{l} \mathrm{U} / \mathrm{ml})$ & $1.68 \pm 0.9$ & $2.07 \pm 1.4$ & $p=0.016$ & $1.40 \pm 0.7$ & 0.016 & $<0.001$ \\
\hline
\end{tabular}


Table 2 Changes $(\Delta)$ in thyroid axis hormone concentrations as a function of psychiatric medication used in treatment of acute psychosis, Mean \pm SD $(N=86)$

\begin{tabular}{lllll}
\hline $\begin{array}{l}\text { Psychiatric } \\
\text { medication }\end{array}$ & & & $\mathbf{t}$ & $\begin{array}{l}\mathbf{p}^{*} \text { (unpaired } \\
\text { t-test) }\end{array}$ \\
\hline Antidepressants & Used & Not used & & \\
& $\mathrm{N}=25$ & $\mathrm{~N}=61$ & & \\
$\Delta \mathrm{FT3}$ (pmol/l) & $0.42 \pm 0.96$ & $0.76 \pm 1.31$ & 0.425 & 0.675 \\
$\Delta \mathrm{FT4}$ & $0.47 \pm 2.77$ & $1.41 \pm 3.43$ & 2.138 & 0.074 \\
$\Delta \mathrm{TSH}$ & $-0.28 \pm 1.82$ & $-0.43 \pm 1.3$ & -0.598 & 0.556 \\
Antipsychotics & Typical & Atypical & & \\
(Typical vs atypical) & $\mathrm{N}=42$ & $\mathrm{~N}=44$ & & \\
$\Delta \mathrm{FT3}$ & $0.85 \pm 1.15$ & $0.49 \pm 1.27$ & 0.507 & 0.813 \\
$\Delta \mathrm{FT4}$ & $1.09 \pm 3.49$ & $1.18 \pm 3.07$ & 1.736 & 0.086 \\
$\Delta \mathrm{TSH}$ & $-0.44 \pm 1.55$ & $-0.34 \pm 1.39$ & -0.335 & 0.738 \\
\hline
\end{tabular}

*Controlled according age (years), gender.

\section{Discussion}

In this study we have found significant changes in thyroid axis hormone concentrations after treatment for acute psychotic episode with antipsychotic drugs. We confirmed that antipsychotic treatment results in recuperation of euthyroid hyperthyroxinemia that was present at the time of admission. However, the decrease in FT4 concentrations was associated with less improvement in psychotic symptom severity. In addition, we have reported the novel finding that antipsychotic treatment resulted in decrease of mean FT3 concentrations and in increase of mean TSH concentrations.

Our finding that FT4 concentrations decreased to normal level after antipsychotic treatment is in line with earlier findings of others regarding total T4 concentrations [18,24]. For example, Kelly and Conley [24] assessed thyroid function in 38 adult treatment-resistant schizophrenic

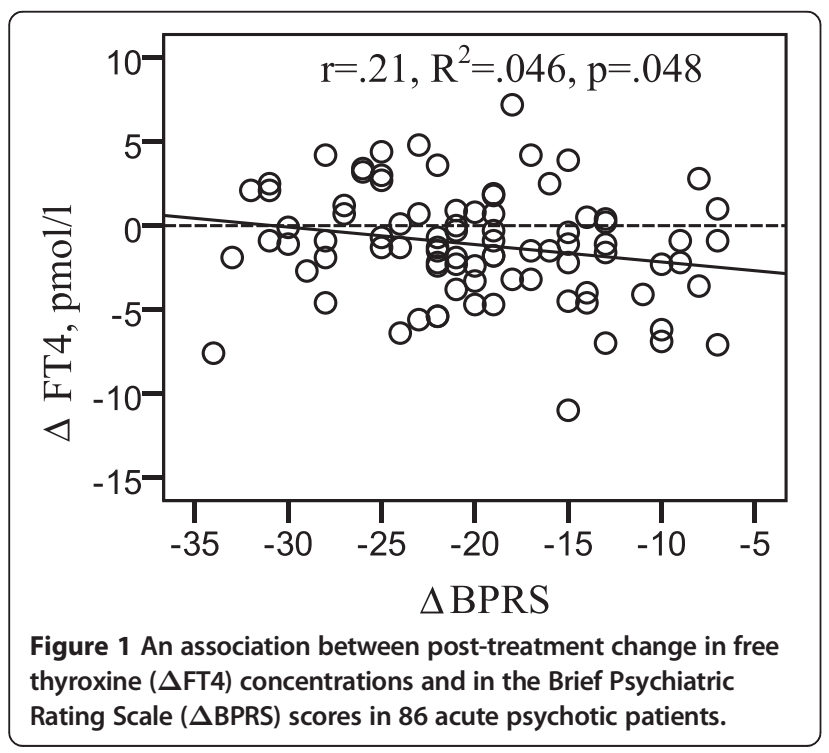

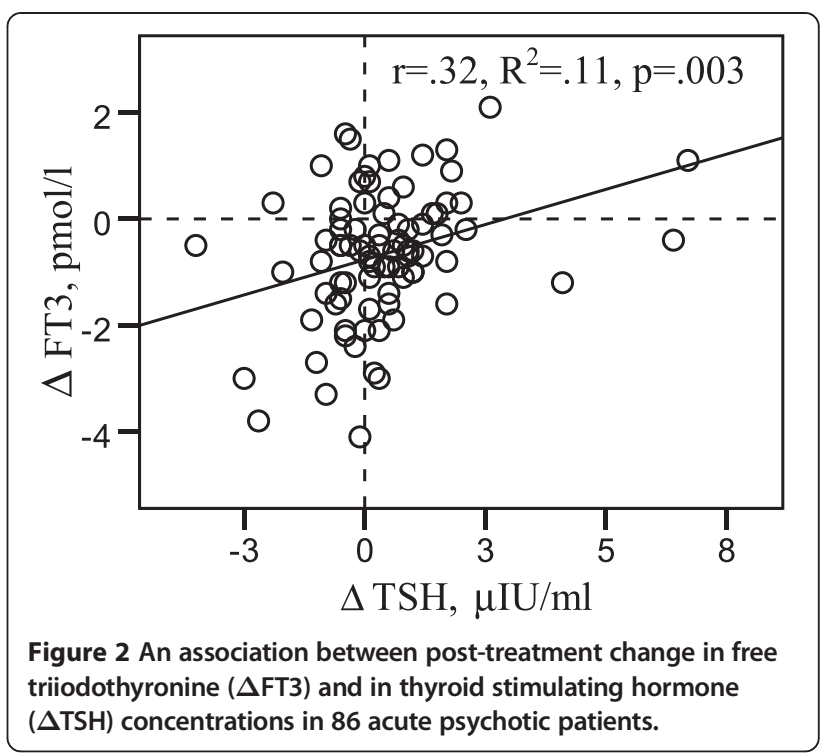

patients after six weeks of treatment with various antipsychotics. They have found little change in thyroid axis function, with an exception of a significant decrease in total T4 serum concentrations in patients taking quetiapine. The authors suggested that the decrease in total $\mathrm{T} 4$ concentrations during quetiapine treatment may be related to competitive metabolism of thyroid hormones and quetiapine by liver enzymes. In our study, the effect of quetiapine (seven patients) on thyroid axis hormone concentrations was similar to other antipsychotic drugs. We think that in the drug resistant patients studied by Kelly and Conley [24] sluggishness of thyroid axis function to respond to antipsychotic drugs may have been an endocrine expression of treatment resistance.

In the studies of Baumgartner et al. [18] and Kelly and Conley [24] significant changes were confined to T4 concentrations. Changes in other thyroid axis hormone concentrations were not significant. By contrast, in the present study significant changes in T3 and TSH concentrations were also noted. This apparent inconsistency may be explained, at least in part, by our larger sample size allowing to reveal an array of dynamics between the thyroid axis variables and mental state.

Regarding all three thyroid axis hormones that we measured, changes in their concentrations after treatment were mostly predicted by concentrations at baseline. It is important to note that changes were not limited to decrease of high concentrations; in a significant proportion of patients increase of low concentrations occurred. Taken together, these two sets of findings suggest stabilization of thyroid axis function after recovery from acute psychosis. The clinical meaning of such thyroid hormone stabilizing effect needs clarification. It may be explained as a simple regression to the mean, the mean having been skewed by the event of acute psychosis. However, if regression to the mean were the only dynamic at work, values of all three 

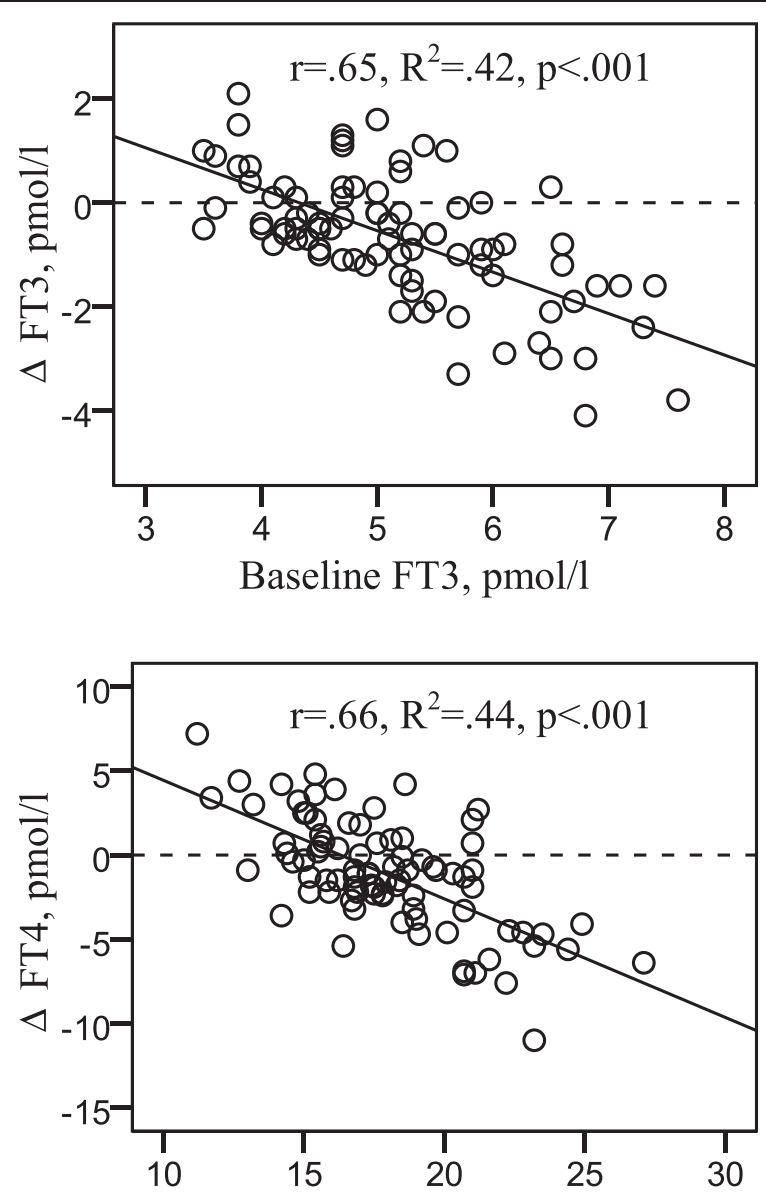

Baseline FT4, pmol/1

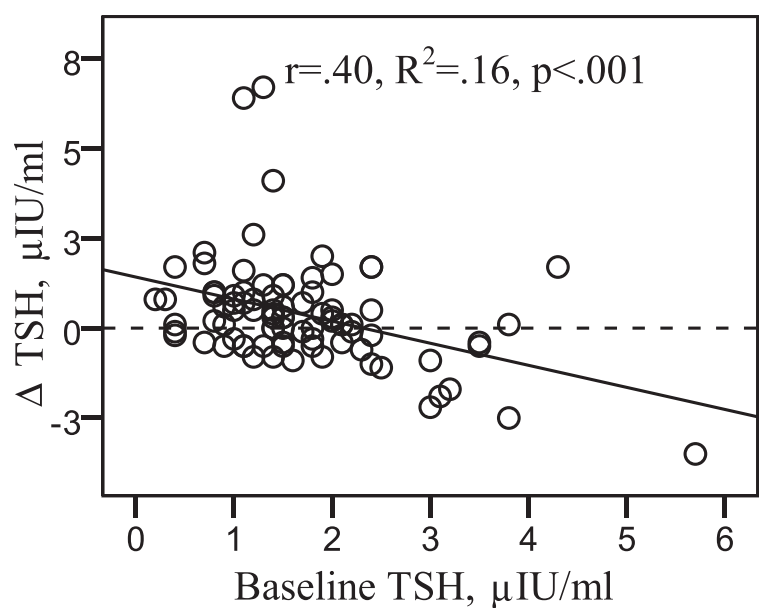

Figure 3 Associations between baseline and post-treatment change $(\Delta)$ in free triiodothyronine (FT3), free thyroxine (FT4) and thyroid stimulating hormone (TSH) concentrations in 86 acute psychotic patients.

hormones studied should have behaved in consistent fashion. The notion of regression to the mean fits well for FT4 concentrations but not for FT3 and TSH concentrations. When compared to healthy blood donors, in psychotic patients mean FT4 concentrations being high before treatment returned to the normal range after treatment. In contrast, mean FT3 concentrations, being in the normal range before treatment, decreased significantly and fell below the 
Table 3 Multivariate linear regression models with changes $(\Delta)$ in thyroid axis hormones concentrations as dependent variables ( $\beta$-values)

\begin{tabular}{|c|c|c|c|}
\hline & $\begin{array}{l}\Delta \text { Free } \\
\text { triiodothyronine }\end{array}$ & $\begin{array}{l}\Delta \text { Free } \\
\text { thyroxine }\end{array}$ & $\begin{array}{l}\Delta \text { Thyroid } \\
\text { stimulating } \\
\text { hormone }\end{array}$ \\
\hline Gender (men/women) & -.262 & -.068 & -.028 \\
\hline Age (years) & -.108 & -.064 & .046 \\
\hline $\begin{array}{l}\text { Duration of hospitalization } \\
\text { (days) }\end{array}$ & -.146 & .048 & .069 \\
\hline $\begin{array}{l}\text { Number of past psychotic } \\
\text { episodes }\end{array}$ & .081 & .241 & -.026 \\
\hline $\begin{array}{l}\text { Duration of the disease } \\
\text { (years) }\end{array}$ & .157 & -.077 & -.083 \\
\hline $\begin{array}{l}\text { Baseline Brief Psychiatric } \\
\text { Rating Scale (score) }\end{array}$ & $.152^{*}$ & .167 & .095 \\
\hline $\begin{array}{l}\text { Antidepressant treatment } \\
\text { (no/yes) }\end{array}$ & .058 & .008 & .066 \\
\hline $\begin{array}{l}\text { Benzodiazepine treatment } \\
\text { (no/yes) }\end{array}$ & .025 & .046 & -.023 \\
\hline $\begin{array}{l}\text { Antipsychotic used } \\
\text { (atypical/ typical) }\end{array}$ & -.083 & .220 & .050 \\
\hline $\begin{array}{l}\text { Antipsychotics use upon } \\
\text { hospitalization (no/yes) }\end{array}$ & -.119 & $.186^{*}$ & -.028 \\
\hline $\begin{array}{l}\text { Baseline respective } \\
\text { hormone concentration }\end{array}$ & -.749 & -.604 & -.399 \\
\hline$R^{2}$ & 0.48 & 0.51 & 0.06 \\
\hline
\end{tabular}

normal concentrations after treatment. TSH concentrations after treatment remained significantly higher in psychotic patients relative to the control group. This assembly of effects suggests that more than regression to the mean was involved; it may also suggest direct effects of antipsychotic

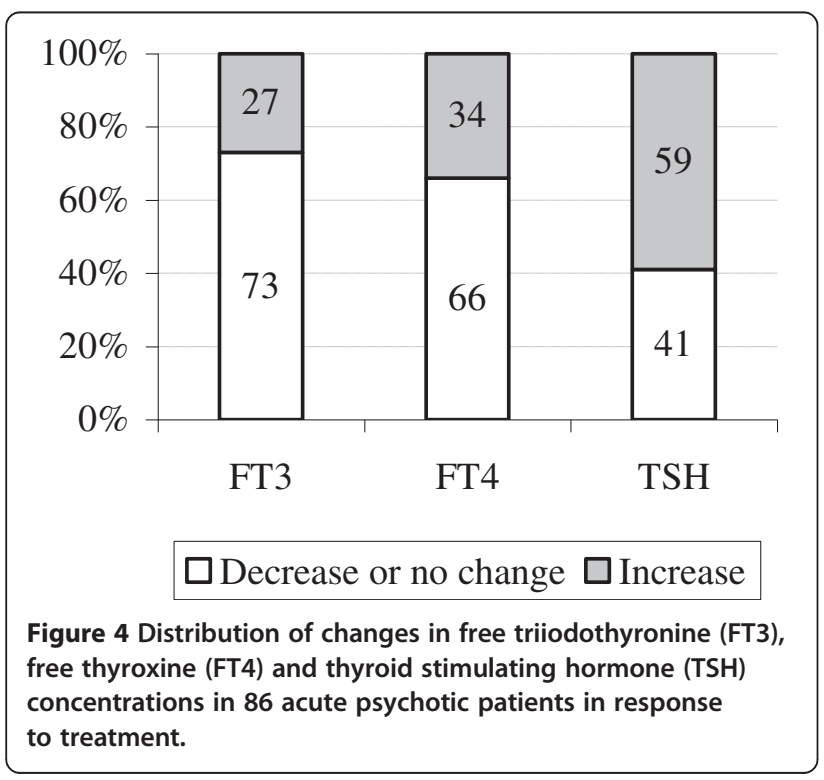

drugs. A similar conclusion was reached by Baumgartner et al. [18] that thyroid function may be altered as a direct consequence of antipsychotic drug treatment.

After evaluating a relationship of changes in thyroid axis hormone concentrations with changes in symptom severity we did not replicate findings of Baumgartner and colleagues [18] who have reported that thyroid hormone concentrations before treatment predicted treatment response. In our study, improvement in BPRS scores was negatively, not positively, associated with the magnitude of changes in FT4 concentrations; the more FT4 concentrations decreased, the less improvement in mental symptoms was achieved. These discrepancies may be explained by some methodological differences between the two studies. Baumgartner et al. [18] measured total T4 concentrations (most of which is bound) while we measured free T4. Gabay and Kushner [25] reported that thyroid hormone binding proteins are acute phase proteins. Inflammation plays a role in acute psychosis [26] and may affect protein bound hormone concentrations. Therefore, measurement of total T4 concentrations may capture immune as well as endocrine changes, while free T4 is more likely to reflect endocrine changes unalloyed by co-existing immune processes.

In the present study, the fall of FT4 concentrations, but not FT3 concentrations, was associated with worse treatment outcome. Low T3 concentration is a laboratory manifestation of the sick euthyroid syndrome that is prevalent in psychiatric patients [27,28]. Thyroid hormone concentrations, including a manifestation of the sick euthyroid syndrome, are associated with genetic polymorphisms of thyroid axis enzymes involved in the metabolism of thyroid hormones. For example, a study led by Cooper-Kazas et al. [28] has found that low T3 concentrations in patients with major depression were associated with polymorphism in the DIO1 gene encoding type-1 deidionase, an enzyme responsible for conversion of $\mathrm{T} 4$ to the more active $\mathrm{T} 3$ in peripheral tissues. Since our study provided no genetic data, we can only speculate that genetic polymorphism in thyroid axis proteins, such as DIO1, and/or elevated activity of type-1 deidionase may have partially accounted for the observed findings regarding the association of FT3 and FT4 concentrations with treatment outcome.

Considering how antipsychotic drugs may exert their effects on the thyroid axis we must enter the caveat that we cannot separate drugs effects from what might be called simply the effects of improvement because almost all our patients improved and all had taken drugs. Inspection of Figure 3 shows that none of the endocrine variables measured changed in the same way, or even in the same direction. Clearly there are subgroups. In a main, our patients overtime decreased concentrations of thyroid hormones and increased TSH concentrations. One could simply say that drug treatment damped hyperthyroxinemia caused by psychosis and that this in turn diminished the 
negative feedback on pituitary gland, allowing TSH to rise. On the other hand we have found a positive correlation between change in FT3 concentrations and change in TSH concentrations, suggesting other effects of antipsychotics on thyroid axis function.

At the cellular level a classical effect of antipsychotics is to block the action of the dopamine. This blockade may have consequences for the thyroid axis, because dopamine exerts tonic inhibition on pituitary TSH secretion. Thus, antipsychotics blocking the inhibition effects caused by dopamine may enhance TSH secretion. This in turn may stimulate T3 production by the thyroid gland and by peripheral tissues [29]. This arrangement may explain the positive correlation between magnitude of change in FT3 concentrations and magnitude of change in TSH concentrations.

A limitation of our study is the absence of patients without antipsychotic treatment (placebo group), not allowing us to determine if changes in thyroid axis function are caused by treatment with antipsychotics or by remission of psychosis itself. Lack of genetic information on thyroid axis related proteins and morphological assessment of the thyroid gland are another limitations. Finally, the absence of a control procedure in the control subjects precluded from investigating the degree of thyroid hormone variability in the control group subjects. Thus, subsequent studies should consider including a control procedure in the control group subjects in order to demonstrate normal thyroid hormone variability. However, our relatively large sample size allowed us to describe thyroid axis hormone variation patterns in response to treatment with antipsychotics. Our finding that such a treatment results in a decrease in FT3 concentrations is new and needs attention. Low T3 concentrations were reported in depressed patients [8], and compensation of this syndrome with exogenous $\mathrm{T} 3$ resulted in augmentation of antidepressive response at least in patients with $D I O 1$ polymorphism [28].

\section{Conclusions}

The study indicates a decrease in mean FT3 concentrations and an increase in mean TSH concentrations after recovery from acute psychosis. Symptomatic improvement is less expressed in patients who experience a decrease in FT4 concentrations.

\section{Competing interests}

RB has received grants/research support, consulting fees and honoraria within the last 5 years from Lundbeck, AstraZeneca, Teva, Glaxo. VS has received the research support and honoraria within the last 5 years from Pfizer, Servier, Sanofi-Aventis and Johnson \& Johnson. AJP declare he has have no competing interests.

\section{Authors' contributions}

RB and AJP designed the study and wrote the protocol, edited manuscript. VS managed the literature searches, statistical analysis, performed psychiatric evaluations and wrote the first draft of the manuscript. All authors contributed to and approved the final manuscript.

\section{Authors' information}

R.B. is MD., PhD, psychiatrist, the director of Behavioral Medicine Institute, Lithuanian University of Health Sciences. V.S is MD., PhD, psychiatrist, lecturer in Clinic of Psychiatry, Lithuanian University of Health Sciences.

\section{Acknowledgements}

Funding for this study was provided by the Lithuanian Fund for Research and Studies; Grant \# T-58/06. This Fund had no further role in study design; in the collection, analysis and interpretation of data; in the writing of the report; and in the decision to submit the paper for publication.

\section{Author details}

'Behavioral Medicine Institute, Lithuanian University of Health Sciences, Vyduno str. 4, Palanga LT-00135, Lithuania. ${ }^{2}$ Clinic of Psychiatry, Lithuanian University of Health Sciences, Mickeviciaus str. 9, Kaunas LT-44307, Lithuania. ${ }^{3}$ Department of Psychiatry, School of Medicine, University of North Carolina at Chapel Hill, Chapel Hill, NC, USA.

Received: 10 April 2014 Accepted: 26 September 2014

Published online: 08 October 2014

\section{References}

1. van Os J, Kapur S: Schizophrenia. Lancet 2009, 374:635-645

2. Moller HJ: Pharmacotherapy of schizophrenic patients: achievements, unsolved needs, future research necessities. Curr Pharm Biotechnol 2012, 13:1476-1489.

3. Bunevicius R, Prange AJ Jr: Thyroid disease and mental disorder: cause and effects or only comorbidity? Curr Opin Psychiatr 2012, 23:363-368.

4. Tandon R, Halbreich U: The second-generation 'atypical' antipsychotics: similar improved efficacy but different neuroendocrine side effects. Psychoneuroendocrinology 2003, 28:1-7.

5. Sim K, Chong SA, Chan YH, Lum WM: Thyroid dysfunction in chronic schizophrenia within a state psychiatric hospital. Ann Acad Med Singapore 2002, 31:641-644.

6. Yazici $K$, Yazici AE, Taneli B: Different neuroendocrine profiles of remitted and nonremitted schizophrenic patients. Pro Neuropsychopharmacol Biol Psychiatr 2002, 26:579-584.

7. Roca RP, Blackman MR, Ackerley MB, Harman SM, Gregerman RI: Thyroid hormone elevations during acute psychiatric illness: relation-ship to severity and distinction from hyperthyroidism. Endocr Res 1990, 16:415-447.

8. Premachandra BN, Kabir MA, Williams IK: Low T3 syndrome in psychiatric depression. J Endocrinol Invest 2006, 29:568-572.

9. Bauer M, Goetz T, Glenn T, Whybrow PC: The thyroid-brain interaction in thyroid disorders and mood disorders. J Neuroendocrino/ 2008, 20:1101-1114.

10. Khemka D, Ali JA, Koch CA: Primary hypothyroidism associated with acute mania: case series and literature review. Exp Clin Endocrinol Diabetes 2011, 119:513-517.

11. Steibliene V, Mickuviene N, Prange AJ Jr, Bunevicius R: Thyroid axis hormone concentrations in psychotic patients upon hospital admission: effects of prior drug use. Medicina (Kaunas) 2012, 48:229-234.

12. Spratt DI, Pont A, Miller MB, McDougal IR, Bayer MF, McLaughlin WT: Hyperthyroxinemia in patients with acute psychiatric disorders. Am J Med 1982, 73:41-48.

13. Chopra IJ, Solomon DH, Huang TS: Serum thyrotrophin in hospitalised psychiatric patients: evidence for hyperthyrotropinemia as measured by an ultrasensitive thyrotrophin assay. Metabolism 1990, 39:538-543.

14. Prange AJ Jr, Loosen PT, Wilson IC, Meltzer HY, Fang VS: Behavioral and endocrine responses of schizophrenic patients to TRH (pro-tirelin). Arch Gen Psychiatry 1979, 36:1086-1093.

15. Baumgartner A, Graf KJ, Kurten I, Meinhold H: The hypothalamic-pituitarythyroid axis in psychiatric patients and healthy subjects: Parts 1-4. Psychiatry Res 1988, 24:271-332.

16. Rinieris P, Christodoulou GN, Souvatzoglou A, Koutras DA, Stefanis C: Freethyroxine index in schizophrenic patients before and after neuroleptic treatment. Neuropsychobiology 1980, 6:29-33.

17. Southwick S, Mason JW, Giller EL, Kosten TR: Serum thyroxine change and clinical recovery in psychiatric inpatients. Biol Psychiatry 1989, 25:67-74. 
18. Baumgartner A, Pietzcker A, Gaebel W: The hypothalamic-pituitary-thyroid axis in patients with schizophrenia. Schizophr Res 2000, 44:233-243.

19. American Psychiatric Association: DSM-IV-TR: Diagnostic and Statistical Manual of Mental Disorders. In Text Revision. Fourthth edition. Washington: American Psychiatric Association; 2000.

20. Sheehan DV, Lecrubier Y, Sheehan KH, Amorim P, Janavs J, Weiller E, Hergueta T, Baker R, Dunbar GC: The Mini-International Neuropsychiatric Interview (M.I.N.I.): the development and validation of a structured diagnostic psychiatric interview for DSM-IV and ICD-10. J Clin Psychiatry 1998, 59:22-33. quiz 34-57.

21. Spitzer RL, Williams JB, Gibbon M, First MB: The Structured Clinical Interview for DSM-III-R (SCID): I. History, rationale, and description. Arch Gen Psychiatry 1992, 49:624-629.

22. Robins LN, Wing J, Wittchen HU, Helzer JE, Babor TF, Burke J, Farmer A, Jablenski A, Pickens R, Regier DA: The Composite International Diagnostic Interview. An epidemiologic Instrument suitable for use in conjunction with different diagnostic systems and in different cultures. Arch Gen Psychiatry 1998, 45:1069-1077.

23. Mortimer AM: Symptom rating scales and outcome in schizophrenia. BrJ Psychiatry Suppl 2007, 50:57-s14.

24. Kelly DL, Conley RR: Thyroid function in treatment-resistant schizophrenia patients treated with quetiapine, risperidone, or fluphenazine. J Clin Psychiatry 2005, 66:80-84.

25. Gabay C, Kushner I: Acute-phase proteins and other systemic responses to inflammation. N Engl J Med 1999, 340:448-454.

26. Mayilyan KR, Weinberger DR, Sim RB: The complement system in schizophrenia. Drug News Perspect 2008, 21:200-210

27. Bunevicius R: Thyroid disorders in mental patients. Curr Opin Psychiatr 2009, 22:391-395.

28. Cooper-Kazaz R, van der Deure WM, Medici M, Visser TJ, Alkelai A, Glaser B, Peeters RP, Lerer B: Preliminary evidence that a functional polymorphism in type 1 deiodinase is associated with enhanced potentiation of the antidepressant effect of sertraline by triiodothyronine. J Affect Disord 2009, 116:113-116.

29. Santos NC, Costa P, Ruano D, Macedo A, Soares MJ, Valente J, Pereira AT, Azevedo MH, Palha JA: Revisiting thyroid hormones in schizophrenia. J Thyroid Res 2012. Epub 2012 Mar 26.

doi:10.1186/s12888-014-0279-7

Cite this article as: Bunevicius et al: Thyroid axis function after inpatient treatment of acute psychosis with antipsychotics: a naturalistic study. BMC Psychiatry 2014 14:279.

\section{Submit your next manuscript to BioMed Central and take full advantage of:}

- Convenient online submission

- Thorough peer review

- No space constraints or color figure charges

- Immediate publication on acceptance

- Inclusion in PubMed, CAS, Scopus and Google Scholar

- Research which is freely available for redistribution 